\title{
Prognostic analysis of gastric gastrointestinal stromal tumor with synchronous gastric cancer
}

\author{
Mi Lin, Jian-Xian Lin, Chang-Ming Huang ${ }^{*}$, Chao-Hui Zheng, Ping Li, Jian-Wei Xie, Jia-Bin Wang and Jun Lu
}

\begin{abstract}
Background: Many patients with gastric gastrointestinal stromal tumor (GIST) and synchronous gastric cancer have been described, most in single case studies. We retrospectively investigated the clinicopathologic features and prognostic effects of gastric GIST in patients with synchronous gastric cancer.

Methods: The study enrolled 170 patients with gastric GIST, who had undergone complete surgical resection (R0) from January 2000 to December 2011. Forty-two patients had synchronous gastric cancer (CA Group), whereas 128 did not (Non-CA Group). The clinicopathologic features and potential prognostic factors in the two groups were compared.

Results: Patients in the CA Group had more obvious symptoms, but a lower rate of preoperative diagnosis of gastric GIST $(P<0.05)$. The two groups differed significantly in gender, age, greatest tumor diameter, risk stratification, tumor-associated ulcers, and CD117 and CD34 expression $(P<0.05$ each). Univariate analysis showed that age, risk stratification, postoperative oral imatinib and synchronous gastric cancer were predictive factors of survival $(P<0.05)$. Cox regression analysis showed that risk stratification, postoperative oral imatinib and synchronous gastric cancer were independent predictors of survival $(P<0.05)$. Stratified analysis showed that the 5 -year overall survival rate was lower in patients with synchronous gastric cancer than in those without synchronous gastric cancer.
\end{abstract}

Conclusions: Gastric GIST with synchronous gastric cancer had a lower rate of preoperative diagnosis, with correct diagnosis often missed. Survival, however, depended primarily on the gastric cancer.

Keywords: Gastrointestinal stromal tumor, Synchronous gastric cancer, Risk stratification, Prognosis

\section{Background}

Gastrointestinal stromal tumor (GIST) is the most common mesenchymal tumor of the gastrointestinal tract, with the most frequent site being the stomach. Since the first report of synchronous epithelial and stromal tumors in the stomach in 2000, [1] many patients with gastric GIST and synchronous gastric cancer have been described, most in single case studies [2-8]. However, little is known about the synchronous GIST and gastric cancer. Its clinicopathologic characteristics and prognostic factors are unclear. We therefore retrospectively compared clinicopathologic findings and prognostic factors in patients with primary GIST with those in patients with primary GIST and synchronous gastric cancer.

\footnotetext{
*Correspondence: hcmlr2002@163.com

Department of Gastric Surgery, Fujian Medical University Union Hospital, No.29 Xinquan Road, Fuzhou 350001, Fujian Province, China
}

\section{Methods}

Between January 2000 and December 2011, 194 patients diagnosed with primary gastric GIST underwent surgical treatment at the Affiliated Union Hospital of Fujian Medical University, Fuzhou, China. Patients were included if their diagnosis of GIST was confirmed pathologically after surgery and if they underwent initial complete surgical resection (R0) for GIST and/or gastric cancer at our hospital. Patients were excluded if they had malignancies other than gastric cancer along with gastric GIST; if they had distant metastases before surgery; or if their pathological diagnosis was incomplete. Of the 170 patients enrolled, 42 had synchronous gastric cancer (CA Group), and 128 did not (Non-CA Group).

Combinations of abdominal ultrasonography, computed tomography/magnetic resonance imaging, gastroscopy/endoscopic ultrasound were used for diagnosis of
C Biomed Central

(c) 2014 Lin et al.; licensee BioMed Central Ltd. This is an open access article distributed under the terms of the Creative Commons Attribution License (http://creativecommons.org/licenses/by/2.0), which permits unrestricted use, distribution, and reproduction in any medium, provided the original work is properly cited. 
GIST/gastric cancer and for assessment of resectability. Metastatic disease was evaluated by computed tomography of the thorax, abdomen and pelvis and/or chest radiography. The surgical resection (enucleation, wedge resection, segmental resection and total/subtotal organ resection) of the GIST was performed according to the tumor site and size. All patients with gastric cancer underwent a D2 lymphadenectomy as described by the Japanese Classification of Gastric Carcinoma (JCGC) [9]. The risk stratification of GIST was according to the proposed modification of the NIH consensus classification for GIST [10]. The TNM stage of gastric cancer was based on the 7th edition of UICC/TNM system [11]. Patients classified as intermediate risk or high risk were suggested to receive $400 \mathrm{mg}$ of imatinib orally after the operation, taken once daily with food, in the form of 100-mg capsules. The therapy was usually given for about 2 years for the intermediate risk and 3 years for the high risk.

The patients were followed up by trained investigators by mail, email, telephone, visits to patients or consultations at the outpatient clinic. The last follow-up date was February 2013. Survival duration was defined as the interval between the date of operation to the date of last contact, date of death, or date on which survival information was collected (due, for example, to loss of contact or death from other causes).

\section{Statistical analysis}

All statistical analysis was performed using the Statistical Package for the Social Sciences (SPSS), version 18.0 for Windows (SPSS Inc, Chicago, USA). Measurement data were reported as means \pm standard deviations, while enumerated data were assessed using the Chi-square or Fisher's exact test. Kaplan-Meier curves were used to estimate overall survival time, with univariate comparisons between groups through the log-rank test. Multivariate analysis using the Cox model was used to evaluate independent predictors of survival. A $P$ value $<0.05$ was considered statistically significant.

\section{Ethical approval}

Ethics Committee of Fujian Medical University Union Hospital approved this retrospective study. Written consent was given by the patients for their information to be stored in the hospital database and used for research.

\section{Results}

\section{Clinicopathologic features}

In the 170 patients, there were 93 males and 77 females, with a male to female ratio of 1.21:1. The mean age at diagnosis of GIST was $61.1 \pm 12.0$ years. For GIST, 52 patients were classified as very low risk, 58 as low risk, 29 as intermediate risk, and 31 as high risk. In the CA Group, the staging of the synchronous gastric cancer was as follows: 14 patients were classified as Stage IA, 8 as Stage IB, 5 as Stage IIA, 1 as Stage IIB, 7 as Stage IIIA, 4 as Stage IIIB, and 3 as Stage IIIC. The histological subtype of the gastric cancer was as follows: 6 patients were classified as well differentiated, 21 as moderately differentiated, 10 as poorly differentiated and 5 as signet ring cell (SRC) histology. Compared with the Non-CA Group, the CA Group had a higher percentage of males, was older in age, and had a lower frequency of ulcer, a smaller greatest tumor diameter, lower risk stratification, and lower positivity rates for CD117 and CD34, with all of these differences being statistically significant (Table 1 ).

\section{Diagnosis}

Of the 146 (85.9\%) symptomatic patients, 97 had abdominal pain, 38 had abdominal tenderness, 33 had black stool, 32 had abdominal distension, 30 had weight loss, 18 had eructation, 16 had anorexia, 16 had sour regurgitation, 16 had hematemesis, 14 had an abdominal mass, 11 had a loss of strength, 11 had dysphagia, 7 had vomiting and 6 had nausea. The proportion of patients with symptoms was significantly higher in the CA than in the Non-CA Group $(P<0.05)$ (Table 1$)$. Of the 97 patients preoperatively diagnosed with gastric GIST, 50 were diagnosed by computed tomography, 38 by abdominal ultrasonography, 8 by magnetic resonance imaging, 2 by gastroscopy, and 37 by endoscopic ultrasound, while 8 patients were confirmed to have the disease by endoscopic biopsy pathology. GISTs in the remaining 73 patients were detected incidentally during surgery or by postoperative analysis of resected specimens, with patients being subsequently diagnosed with gastric GIST by postoperative pathology. Of the 128 patients in the Non-CA group, 88 tumors $(68.8 \%)$ were identified before surgery but not confirmed by pathology, 8 (6.3\%) were confirmed before surgery and $32(25.0 \%)$ were confirmed after surgery. In the CA group, however, only 1 tumor (2.4\%) was detected before surgery, whereas 41 (97.6\%) were confirmed after surgery. The rate of preoperative diagnosis was significantly lower in the CA than in the Non-CA Group (2.4\% versus $97.6 \%, P=0.000$ ) (Table 2 ).

\section{Long-term surgical outcomes}

Of the 170 patients, 165 (97.1\%) were followed up for 2 to 127 months (median, 38 months), including 40 patients (95.2\%) in the CA Group and 125 (97.7\%) in the Non-CA Group. During follow-up, 23 patients died, 14 in the CA and 9 in the Non-CA Group. The 3- and 5 -year overall survival (OS) rates were $87.0 \%$ and $82.3 \%$, respectively, for the entire cohort, $62.6 \%$ and $57.8 \%$, respectively, for the CA group, and $94.8 \%$ and $90.1 \%$, respectively, for the Non-CA group. The between-group differences were statistically significant (Figure 1). 
Table 1 Clinicopathologic features of all patients (cases (\%))

Items

Male/Female

Age(yr)

$\leq 60 />60$

Tumor location

Upper

Middle

Lower

Greatest tumor diameter $(\mathrm{cm})$

$\leq 2$

From $>2$ to 5

From $>5$ to 10

$>10$

Tumor bleeding

Yes/No

Tumor ulceration

Yes/No

Mitotic count

$\leq 5 / 50 \mathrm{HPF}$

From $>5$ to $10 / 50 \mathrm{HPF}$

$>10 / 50$ HPF

Risk stratification

Very low

Low

Intermediate

High

Tumor necrosis

Yes/No

\section{Cystic tumor}

Yes/No

CD117

$(-) /(+)$

CD34

$(-) /(+)$

SMA

$(-) /(+)$

S-100

$(-) /(+)$

Postoperative complications

Yes/No

Non-CA Group

61(47.7)/67(52.3)

75(58.6)/53(41.4)

61(47.7)

45(35.2)

22(17.2)

19(14.8)

69(53.9)

24(18.8)

16(12.5)

15(11.7)/113(88.3)

36(28.6)/92(71.4)

97(75.8)

21(16.4)

10(7.8)

17(13.3)

54(42.2)

27(21.1)

30(23.4)

$7(5.5) / 121(94.5)$

$10(.8) / 118(92.2)$

19(14.8)/109(85.2)

11(8.6)/117(91.4)

$82(64.1) / 46(35.9)$

116(90.6)/12(9.4)

18(14.1)/110(85.9)

\begin{tabular}{ll} 
CA Group & $P^{*}$ \\
\hline $0.001^{*}$
\end{tabular}

$32(76.2) / 10(23.8)$

$0.001^{*}$

12(28.6)/30(71.4)

14(33.3)

20(47.6)

8(19.0)

$0.000^{*}$

35(83.3)

7(16.7)

$0(0.0)$

$0(0.0)$

1(2.4)/41(97.6)

$0.014^{*}$

$4(9.5) / 38(90.5)$

38(90.5)

$3(7.1)$

1(2.4)

$0.000^{*}$

35(83.3)

4(9.5)

2(4.8)

1(2.4)

1(2.4)/41(97.6)

$0(0.0) / 42(100.0)$

$0.009^{*}$

14(33.3)/28(66.7)

$0.000^{*}$

17(40.5)/25(59.5)

$30(71.4) / 12(28.6)$

$38(90.5) / 4(9.5)$

$9(21.4) / 33(78.6)$

${ }^{*}$ Analysis of variance. $P<0.05$ is significant. CA Group, gastric GIST patients with synchronous gastric cancer; Non-CA Group, gastric GIST patients without synchronous gastric cancer. 
Table 2 Diagnosis of all patients (cases (\%))

\begin{tabular}{lccc}
\hline Items & Non-CA Group & CA Group & $\mathbf{P}^{*}$ \\
\hline Symptom & & & $0.012^{*}$ \\
Symptomatic & $105(82.0)$ & $41(97.6)$ & \\
Asymptomatic & $23(18.0)$ & $1(2.4)$ &
\end{tabular}

\section{Diagnosis}

Preoperative

96(75.0)

Postoperative

$32(25.0)$

41(97.6)

${ }^{*}$ Analysis of variance. $P<0.05$ is significant. CA Group, gastric GIST patients with synchronous gastric cancer; Non-CA Group, gastric GIST patients without synchronous gastric cancer.

\section{Univariate and multivariate survival analysis}

Univariate analysis showed that patient age, risk stratification, postoperative oral imatinib and synchronous gastric cancer were predictive factors of survival $(P<0.05$; Table 3). Cox regression analysis showed that risk stratification, postoperative oral imatinib and synchronous gastric cancer were independent predictors of OS $(P<0.05$; Table 4).

\section{Survival analysis based on risk stratification}

The 5-year survival rates were significantly lower among patients with synchronous gastric cancer than among patients without synchronous gastric cancer, both among patients stratified as being at very low risk/low risk $(60.2 \%$ versus $98.6 \%, P<0.05)$ and among those stratified as

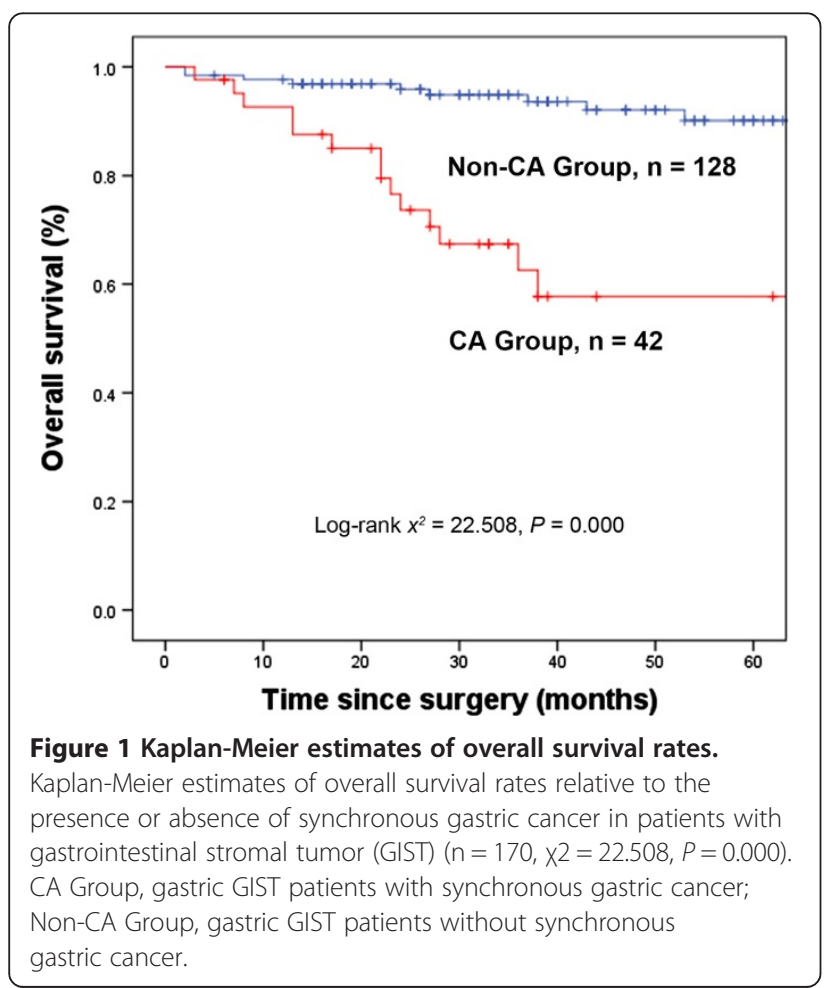

Table 3 Univariate analysis of variables associated with survival in 170 patients with gastric gastrointestinal stromal tumor (GIST)

\begin{tabular}{lll}
\hline Items & $\begin{array}{l}\text { Cases (5-year } \\
\text { survival rate,\%) }\end{array}$ & $P^{*}$ \\
\hline Gender & \\
Male/Female & $93(77.1) / 77(88.2)$
\end{tabular}

Age (yr)

87(89.3)/83(74.2)

Tumor location

Upper/Middle/Lower

$75(84.0) / 65(76.3) / 30(92.1)$

Greatest tumor diameter $(\mathrm{cm})$

$\leq 2 />2$ to $5 />5$ to $10 />10$

54(77.3)/76(92.5)/24(78.4)/ 16(63.3)

Tumor bleeding

Yes/No

16(100.0)/154(80.1)

Tumor ulceration

Yes/No

40(92.3)/130(78.4)

Mitotic count (/50 HPF)

$\leq 5 />5$ to $10 />10$

135(86.1)/24(67.9)/11(70.7)

Risk stratification

Very-low

$0.004^{*}$

Low

$52(76.2)$

Intermediate

$58(94.2)$

29(96.6)

High

$31(58.4)$

CD117

$(-) /(+)$

$33(64.8) / 137(85.8)$

CD34

$(-) /(+)$

$28(71.2) / 142(84.3)$

SMA

$(-) /(+)$

$112(82.2) / 58(82.9)$

S-100

$(-) /(+)$

154(82.3)/16(84.4)

Tumor necrosis

Yes/No

$8(87.5) / 162(81.7)$

Cystic tumor

Yes/No

10(100.0)/160(81.6)

Synchronous gastric cancer

Yes/No

$42(57.8) / 128(90.1)$

SRC in synchronous gastric cancer

Yes/No

$5(40 \%) / 37(59.2)$

Postoperative complications

Yes/No

$27(83.8) / 141(82.2)$

Postoperative oral imatinib

Yes/No

53(97.0)/117(77.3)

$S R C$, signet ring cell.

*Analysis of variance. $P<0.05$ is significant. 
Table 4 Multivariate analysis of factors prognostic of survival in patients with gastric gastrointestinal stromal tumor (GIST) and synchronous gastric cancer

\begin{tabular}{|c|c|c|c|c|c|c|}
\hline Parameters & $\beta$ & SE & Wald & $P^{*}$ & RR & $95 \% \mathrm{Cl}$ \\
\hline Age & -0.596 & 0.481 & 1.531 & & 0.551 & $0.215-1.416$ \\
\hline Synchronous gastric cancer & -2.296 & 0.602 & 14.571 & $0.000^{*}$ & 0.101 & $0.031-0.327$ \\
\hline Risk stratification & & & 24.190 & $0.000^{*}$ & & \\
\hline Very low versus high & -2.504 & 0.607 & 17.001 & $0.000^{*}$ & 0.082 & $0.025-0.269$ \\
\hline Low versus high & -2.544 & 0.682 & 13.895 & $0.000^{*}$ & 0.079 & $0.021-0.299$ \\
\hline Intermediate versus high & -2.638 & 1.060 & 6.191 & $0.013^{*}$ & 0.071 & $0.009-0.571$ \\
\hline Postoperative oral imatinib & 2.213 & 1.045 & 4.489 & $0.034^{*}$ & 9.146 & $1.180-70.864$ \\
\hline
\end{tabular}

*Analysis of variance. $P<0.05$ is significant.

being at intermediate risk/high risk (33.3\% versus $98.1 \%$, $P<0.05$ ) (Figure 2).

\section{Discussion}

The incidence of GIST is only approximately 10 to 20 cases per million per year, [12-16] with gastric GIST being the most common type. Although GISTs are rare, the proportion of GIST patients who present synchronously with other malignancies is not low. In particular, the combination of gastric GIST and synchronous gastric cancer is relatively common. An analysis of 14 studies found that $4.5 \%$ to $33 \%$ of patients had GIST simultaneously with other neoplasms [17]. In our series we found that 42 of $170(24.7 \%)$ patients with gastric GISTs presented with synchronous gastric cancers. Gastric GISTs accompanied by synchronous gastric cancer have specific pathological features. For example, 14 of 15 gastric GISTs with synchronous gastric cancer were smaller than $2.0 \mathrm{~cm}$ in size, with the fifteenth being $2.5 \mathrm{~cm}$; moreover, almost all of these tumors were stratified as very low or low risk [2]. Similarly, we found that most of the gastric GISTs in patients with synchronous gastric cancer were small and of very low or low risk of malignancy. Moreover, only one of the 42 patients (2.4\%) found to have gastric GIST with synchronous gastric cancer was diagnosed preoperatively, with all others detected incidentally during surgery or in postoperative pathology, a finding in agreement with previous results $[1,2,18]$.

Clinical manifestations of gastric GIST were nonspecific, with some patients having no clinical manifestations when the tumor was small. The preoperative diagnosis of GIST depended mainly on imaging modalities, such as computed tomography and endoscopy $[19,20]$. In patients with simultaneous gastric cancer and gastric GIST, the symptoms of gastric GIST were often masked by the clinical symptoms of gastric cancer. Most of these patients had small GISTs $(<2.0 \mathrm{~cm})$ and saw a doctor for the symptoms of gastric cancer. Moreover, since most gastric GISTs were submucosal, muscular, or subserosal, patients often could not be preoperatively diagnosed by endoscopic biopsy. Furthermore, many clinicians lack the knowledge of

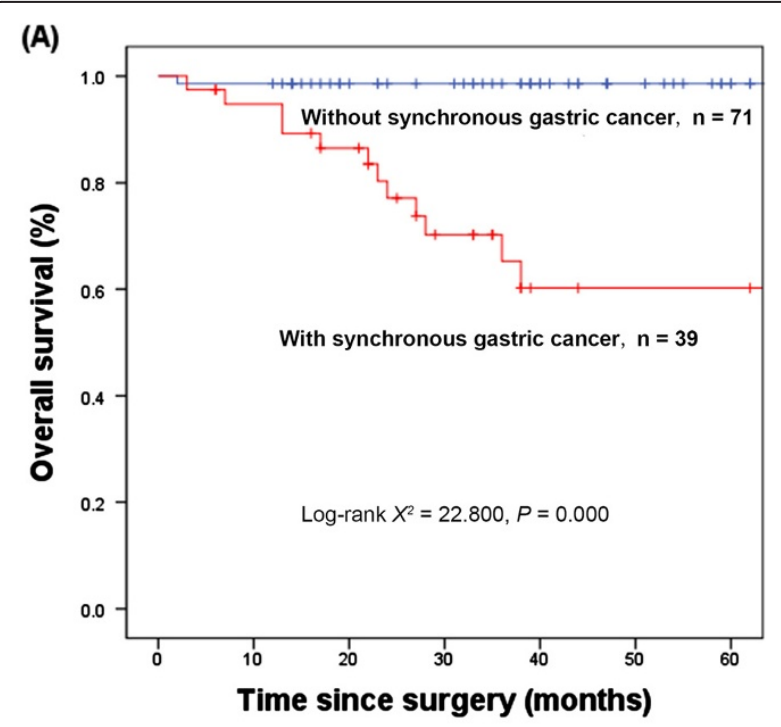

(B)

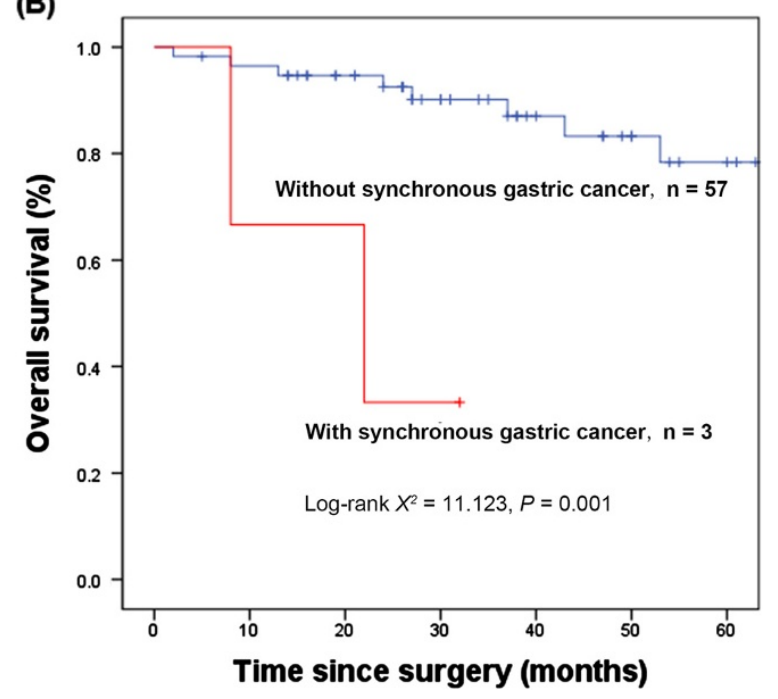

Figure 2 Kaplan-Meier estimates of overall survival rates based on risk stratification. Kaplan-Meier estimates of overall survival rates in gastric gastrointestinal stromal tumor (GIST) patients with and without synchronous gastric cancer and at $(\mathbf{A})$ very low/low risk $(n=110, \times 2=22.800, P=0.000)$ and at $(B)$ intermediate/high risk $(n=60, \times 2=11.123, P=0.001)$. 
multiple primary tumors and are satisfied with a diagnosis of gastric cancer alone, resulting in a low rate of preoperative diagnosis of gastric GIST.

Interestingly, we found that the gastric GIST patients with synchronous gastric cancer were older in age compared with those without synchronous gastric cancer, and that was a predictive factor of survival. However, the age was not an independent predictor of OS. We speculated that the finding might be associated with the high incidence of gastric cancer in older patients. In addition, the elderly might be with some change of gene expression profile and a lower immunity, resulting in more easily suffering from the synchronous tumors. Further studies are needed on the gene expression in primary tumor cells from older and younger patients and signal transduction may also provide us with some clues to this finding.

Common immunohistochemistry included CD117, CD34, SMA, S-100 of GIST were analyzed in our study, where we found statistically different positive rates of CD117 and CD34 between groups. However, further prognosis analysis suggested this finding was not related to prognosis. We found that the gastric GIST with synchronous gastric cancer had a lower positive rate of CD117 and CD34 based on the large sample. This was a finding not encountered before in the literature. It might be worth forming a base of classification of GIST tumors according to it. More research is needed.

Previously, GIST was associated with a poor prognosis, with 5-year OS rates after R0 resection ranging from $28 \%$ to $65 \%,[21-25]$ and another study reporting that patients with gastric GIST had a 5-year OS rate of 42\% [26]. Additional studies, improvements in surgical skill, and the introduction of the molecular targeted drug imatinib have significantly improved the prognosis of patients with GIST, with a study in 2010 reporting a 5-year OS rate in 187 patients with gastric GIST being 75.9\% [27]. Few studies to date have assessed the prognosis of patients with synchronous gastric GIST and gastric cancer. A study of 22 patients with gastric GIST and synchronous gastric cancer who underwent surgical treatment found that the 5 -year OS rate was $57.8 \%$, with a median survival time of 36 months [28]. We found that the 5-year OS rate in patients was significantly lower in gastric GIST patients with than without gastric cancer. Furthermore, risk stratification and the presence of synchronous gastric cancer were independent predictors of survival. The prognosis of gastric GIST patients was reported to be poorer for those with synchronous gastric cancer than for those without synchronous gastric cancer, regardless of risk stratification [4]. Similarly, we found that the 5-year OS rates were significantly lower in patients with synchronous gastric cancer than in those without synchronous gastric cancer, whether patients were stratified into the very low/low risk or intermediate/high risk groups. Thus, because of smaller tumor size, lower risk stratification and lower recurrence risk after complete resection, the prognosis of gastric GIST patients with synchronous gastric cancer was good, with GIST itself having little effect on patient prognosis. The main cause of poor prognosis in these patients was advanced synchronous gastric cancer, suggesting that active treatment of the synchronous gastric cancer would improve long-term survival of these patients.

Some studies revealed that SRC histology was associated with worse survival than non-SRC [29-31]. In our study, there were 5 patients with SRC carcinoma in the synchronous gastric cancer. To study the importance of SRC histology on survival, univariate analysis was done in the gastric GIST patients with SRC or with non-SRC, with a result of no significant differences. But the result might be of limitation of the small sample. Previous reports of the prognosis of patients with SRC were controversial. Some studies reported better 5-year survival rates in SRC than in other cell types in early gastric cancer $[32,33]$. However, others reported no significant differences when the stage of gastric cancer matched [34]. It has also been suggested that SRC histology is an independent predictor of poor prognosis in gastric cancer [29].

All GISTs are regarded as having malignant potential. Moreover, in patients with gastric GIST and synchronous gastric cancer, larger sized GISTs and higher risk stratification were associated with a high recurrence rate and poor prognosis, even after complete resection of the GIST [35]. Consequently, gastric GIST should be removed when incidentally discovered during surgery for gastric cancer; when necessary, targeted therapy should be considered.

\section{Conclusions}

Gastric GIST with synchronous gastric cancer had a lower rate of preoperative diagnosis, with correct diagnosis often missed. Survival, however, depended primarily on the gastric cancer, suggesting that active treatment of the synchronous gastric cancer would improve longterm survival of these patients. Moreover, gastric GISTs should be removed when incidentally discovered during surgery for gastric cancer; when necessary, targeted therapy should be considered.

\section{Consent}

Written informed consent was obtained from the patient for the publication of this report and any accompanying images.

Abbreviations

GIST: gastrointestinal stromal tumor; OS: overall survival; SRC: signet ring cell.

\section{Competing interests}

The authors declare that they have no competing interest. 


\section{Authors' contributions}

$\mathrm{ML}, \mathrm{JXL}$ and $\mathrm{CMH}$ conceived the study and participated in its design and coordination. ML, JXL, PL, JWX, JBW and JL helped to collect data. ML performed the statistical analysis. ML and JXL drafted the manuscript. CMH and $\mathrm{CHZ}$ helped revise the paper critically for important intellectual content. All authors read and approved the final manuscript.

Received: 2 August 2013 Accepted: 18 January 2014

Published: 31 January 2014

\section{References}

1. Maiorana A, Fante R, Cesinaro AM, Fano RA: Synchronous occurrence of epithelial and stromal tumors in the stomach: a report of 6 cases. Arch Pathol Lab Med 2000, 124:682-686

2. Yan Y, Li Z, Liu Y, Zhang L, Li J, Ji J: Coexistence of gastrointestinal stromal tumors and gastric adenocarcinomas. Tumour Biol 2013, 34:919-927.

3. Firat Ö, Caliskan C, Karaca C, SEZAK M, ÖZÜTEMIZ Ö, ERSIN S, GÜLER A Coexistence of gastric cancer and gastrointestinal stromal tumor: report of two cases. Turk J Gastroenterol 2010, 21:302-304.

4. Lee FY, Jan YJ, Wang J, Yu CC, Wu CC: Synchronous gastric gastrointestinal stromal tumor and signet-ring cell adenocarcinoma: a case report. Int I Surg Pathol 2007, 15:397-400.

5. Lin YL, Tzeng JE, Wei CK, Lin CW: Small gastrointestinal stromal tumor concomitant with early gastric cancer: a case report. World J Gastroenterol 2006, 12:815-817.

6. Kleist B, Lasota J, Miettinen M: Gastrointestinal stromal tumor and gastric adenocarcinoma collision tumors. Hum Pathol 2010, 41:1034-1039.

7. Katsoulis IE, Bossi M, Richman PI, Livingstone Jl: Collision of adenocarcinoma and gastrointestinal stromal tumour (GIST) in the stomach: report of a case. Int Semin Surg Oncol 2007, 4:2

8. Yamamoto D, Hamada Y, Tsubota Y, Kawakami K, Yamamoto C, Yamamoto M: Simultaneous development of adenocarcinoma and gastrointestinal stromal tumor (GIST) in the stomach: case report. World J Surg Oncol 2012, 10:1-4

9. Japanese Gastric Cancer Association: Japanese classification of gastric carcinoma: 3rd English edition. Gastric Cancer 2011, 14:101-112.

10. Joensuu $\mathrm{H}$ : Risk stratification of patients diagnosed with gastrointestinal stromal tumor. Hum Pathol 2008, 39:1411-1419.

11. Sobin LH, Wittekind C, Gospodarowicz M: TNM classification of malignant tumors (UICC). 7th edition. New York: Wiley-Blackwell; 2009:73-77.

12. Nilsson B, Bümming P, Meis-Kindblom JM, Odén A, Dortok A, Gustavsson B, Sablinska K, Kindblom LG: Gastrointestinal stromal tumors: the incidence, prevalence, clinical course, and prognostication in the preimatinib mesylate era. Cancer 2005, 103:821-829.

13. Monges G, Bisot-Locard S, Blay JY, Bouvier AM, Urbieta M, Coindre JM, Scoazec JY: The estimated incidence of gastrointestinal stromal tumors in France. Results of PROGIST study conducted among pathologists. Bull Cancer 2010, 97:E16-E22

14. Espinosa I, Lee CH, Kim MK, Rouse BT, Subramanian S, Montgomery K, Varma S, Corless CL, Heinrich MC, Smith KS: A novel monoclonal antibody against DOG1 is a sensitive and specific marker for gastrointestinal stromal tumors. Am J Surg Pathol 2008, 32:210-218.

15. Goettsch WG, Bos SD, Breekveldt-Postma N, Casparie M, Herings $R$ Hogendoorn PC: Incidence of gastrointestinal stromal tumours is underestimated: results of a nation-wide study. Eur J Cancer 2005, 41:2868-2872

16. Tzen CY, Wang JH, Huang YJ, Wang MN, Lin PC, Lai GL, Wu CY, Tzen CY: Incidence of gastrointestinal stromal tumor: a retrospective study based on immunohistochemical and mutational analyses. Digest Dis Sci 2007, 52:792-797.

17. Agaimy A, Wünsch PH, Sobin LH, Lasota J, Miettinen M: Occurrence of other malignancies in patients with gastrointestinal stromal tumors. Semin Diagn Pathol 2006, 23:120-129.

18. Chan CH, Cools-Lartigue J, Marcus VA, Feldman LS, Ferri LE: The impact of incidental gastrointestinal stromal tumours on patients undergoing resection of upper gastrointestinal neoplasms. Can I Surg 2012, 55:366-370.

19. Gold JS, DeMatteo RP: Combined surgical and molecular therapy: the gastrointestinal stromal tumor model. Ann Surg 2006, 244:176-184

20. Nowain A, Bhakta H, Pais S, Kanel G, Verma S: Gastrointestinal stromal tumors: clinical profile, pathogenesis, treatment strategies and prognosis. J Gastroenterol Hepatol 2005, 20:818-824.
21. DeMatteo RP, Lewis JJ, Leung D, Mudan SS, Woodruff JM, Brennan MF: Two hundred gastrointestinal stromal tumors: recurrence patterns and prognostic factors for survival. Ann Surg 2000, 231:51-58.

22. Joensuu H: Sunitinib for imatinib-resistant GIST. Lancet 2006, 368:1303-1304.

23. van der Zwan SM, DeMatteo RP: Gastrointestinal stromal tumor: 5 years later. Cancer 2005, 104:1781-1788.

24. Ng E, Pollock RE, Munsell MF, Atkinson EN, Romsdahl MM: Prognostic factors influencing survival in gastrointestinal leiomyosarcomas. Implications for surgical management and staging. Ann Surg 1992. 215:68-77.

25. Ranchod M, Kempson RL: Smooth muscle tumors of the gastrointestinal tract and retroperitoneum: a pathologic analysis of 100 cases. Cancer 1977, 39:255-262

26. Wong N, Young R, Malcomson R, Nayar A, Jamieson L, Save V, Carey F, Brewster D, Han C, Al-Nafussi A: Prognostic indicators for gastrointestinal stromal tumours: a clinicopathological and immunohistochemical study of 108 resected cases of the stomach. Histopathol 2003, 43:118-126.

27. Huang H, Liu YX, Zhan ZL, Liang H, Wang P, Ren XB: Different sites and prognoses of gastrointestinal stromal tumors of the stomach: report of 187 cases. World J Surg 2010, 34:1523-1533.

28. Liu YJ, Yang Z, Hao LS, Xia L, Jia QB, Wu XT: Synchronous incidental gastrointestinal stromal and epithelial malignant tumors. World J Gastroenterol 2009, 15:2027-2031.

29. Piessen G, Messager M, Leteurtre E, Jean-Pierre T, Mariette C: Signet ring cell histology is an independent predictor of poor prognosis in gastric adenocarcinoma regardless of tumoral clinical presentation. Ann Surg 2009, 250:878-887.

30. Li C, Kim S, Lai JF, Hyung WJ, Choi WH, Choi SH, Noh SH: Advanced gastric carcinoma with signet ring cell histology. Oncol 2007, 72:64-68.

31. Kim JP, Kim SC, Yang HK: Prognostic significance of signet ring cell carcinoma of the stomach. Surg Oncol 1994, 3:221-227.

32. Jiang CG, Wang ZN, Sun Z, Liu FN, Yu M, Xu HM: Clinicopathologic characteristics and prognosis of signet ring cell carcinoma of the stomach: results from a Chinese mono-institutional study. J Surg Oncol 2011, 103:700-703.

33. Kunisaki C, Shimada H, Nomura M, Matsuda G, Otsuka Y, Akiyama H: Therapeutic strategy for signet ring cell carcinoma of the stomach. Br J Surg 2004, 91:1319-1324

34. Zhang M, Zhu G, Zhang H, Gao H, Xue Y: Clinicopathologic features of gastric carcinoma with signet ring cell histology. J Gastrointest Surg Off J Soc Surg Aliment Tract 2010, 14:601-606.

35. Hassan I, Shyyan R, Dozois EJ, Smyrk TC, Okuno SH, Schleck CD, Hodge DO, Donohue JH: Surgically managed gastrointestinal stromal tumors: a comparative and prognostic analysis. Ann Surg Oncol 2008, 15:52-59.

doi:10.1186/1477-7819-12-25

Cite this article as: Lin et al.: Prognostic analysis of gastric gastrointestinal stromal tumor with synchronous gastric cancer. World Journal of Surgical Oncology 2014 12:25.

\section{Submit your next manuscript to BioMed Central and take full advantage of:}

- Convenient online submission

- Thorough peer review

- No space constraints or color figure charges

- Immediate publication on acceptance

- Inclusion in PubMed, CAS, Scopus and Google Scholar

- Research which is freely available for redistribution

Submit your manuscript at www.biomedcentral.com/submit
C Biomed Central 